\title{
Enrichment of a Vasoactive Neuropeptide (Calcitonin Gene Related Peptide) in the Trigeminal Sensory Projection to the Intracranial Arteries
}

\author{
Timothy P. O'Connor and Derek van der Kooy \\ Neurobiology Research Group, Department of Anatomy, University of Toronto, Toronto, Ontario, Canada M5S 1A8
}

Trigeminal sensory innervation of cerebral vessels and the surrounding dura is responsible for most intracranial head pain. Small-diameter fibers containing substance P (Sub P) have been observed in the periadventitia around feline cerebral blood vessels, and it has been suggested that these fibers are the trigeminal substrate for vascular pain associated with cluster and migraine headaches. Calcitonin gene related peptide (CGRP) coexists with Sub $P$ in some of these fibers and with some Sub $P$ containing neurons in the trigeminal ganglion. In addition, a population of trigeminal neurons containing CGRP but not Sub $P$ has been observed. We now report that the population of trigeminal ganglion cells projecting to the cerebral vasculature is enriched in CGRP. containing neurons, and especially in the population of neurons containing CGRP and not Sub $P$.

Using retrograde tracing of fluorescent tracers combined with immunocytochemistry after explant culture, we found approximately $32 \%$ of trigeminal ganglion cells projecting to the cerebral vasculature contained CGRP. Approximately 18 and $17 \%$ of these cells contained Sub P and cholecystokinin (CCK), respectively. The $32 \%$ of ganglion cells projecting to the cerebral vasculature that contain CGRP stands in contrast to the $12 \%$ CGRP positive seen in the population of ganglion cells projecting out to another target (the forehead), and the 21 and $23 \%$ CGRP positive observed in the mandibular branch and entire ganglion, respectively. Sub P and CCK are not enriched in the trigeminal innervation of the vasculature compared with their presence in cells throughout the ganglia. Using double-label immunocytochemistry we found that this CGRP enrichment in the cerebral vasculature projection is due to a population of trigeminal cells that contain CGRP but not Sub P. Trigeminal ganglion cells containing CGRP but not Sub P make up $21 \%$ of the cells projecting to the cerebral vasculature but only $9 \%$ of the cells throughout the ganglia. On the other hand, ganglion cells containing both CGRP and Sub P make up $17 \%$ of the cerebral artery projecting cells and $14 \%$ of the whole ganglia population. CCK also coexists in many of the artery projecting ganglion neurons that contain both CGRP and Sub P. Thus, there is a population of artery projecting trigeminal

Received July 27, 1987; revised Nov. 9, 1987; accepted Nov. 11, 1987

Supported by the Ontario Heart and Stroke Foundation and by a Canadian Heart Foundation Research Traineeship.

Correspondence should be addressed to Timothy O'Connor at the above address.

Copyright $\odot 1988$ Society for Neuroscience $0270-6474 / 88 / 072468-09 \$ 02.00 / 0$ cells (and indeed throughout the ganglion) that contain 3 neuropeptides.

The CGRP enrichment emphasizes the putative functional importance of CGRP in regulating cerebral vasculature tone. Further, it raises questions concerning the underlying developmental processes whereby a peripheral target may help regulate the neuropeptide synthesized in its sensory innervation.

The trigeminal innervation of the large cerebral blood vessels and surrounding dura provides the substrate for pain of intracranial origin (Penfield and McNaughton, 1940; Ray and Wolff, 1940; Wirth and Van Buren, 1971). These projections are thought to carry the nociceptive information during a migraine (Moskowitz, 1984) or cluster headache (Hardebo, 1984). Trigeminal cells innervating the middle cerebral artery and middle meningeal artery in the rat and cat are located predominantly in the ophthalmic and maxillary divisions (Mayberg et al., 1981, 1984; Steiger et al., 1982; O'Connor and van der Kooy, 1986a). The pain information carried by these fibers is usually referred to the forehead and ocular region of the face (Fay, 1932; Penfield and McNaughton, 1940; Ray and Wolff, 1940; Wirth and Van Buren, 1971). This referral is mediated via a central convergence of trigeminal fibers innervating the cerebral arteries and forehead region onto common wide-dynamic-range or nociceptivespecific brain-stem neurons (Davis and Dostrovsky, 1986; Strassman et al., 1986). The referred pain is not mediated by a peripheral branching of processes from single ganglion neurons to the 2 different sensory fields, as these collateralized neurons were shown to be quite rare in the adult (Borges and Moskowitz, 1983; McMahon et al., 1985; O'Connor and van der Kooy, 1986a).

As well as being sensory to the cerebral blood vessels, the trigeminal projection also plays a role in vasomotor tone (McCulloch et al., 1986). Stimulation of the appropriate trigeminal projection dilates extracranial blood vessels (Gazelius and Olgart, 1980; Goadsby et al., 1986) and increases vascular permeability in canine teeth (Gazelius and Olgart, 1980). The peptides most likely responsible for these effects appear to be substance P (Sub P) (Olgart et al., 1977) and calcitonin gene related peptide (CGRP) (Hanko et al., 1985; McCulloch et al., 1986). These peptides have been observed in trigeminal ganglion cells (Lee et al., 1985a, b; Skofitsch and Jacobowitz, 1985) and in neural processes running through the dura (Saito et al., 1987) and the periadventitia of cerebral arteries (Liu-Chen et al., 1983, 1986; Hanko et al., 1985; Wanaka et al., 1986). A decrease in 


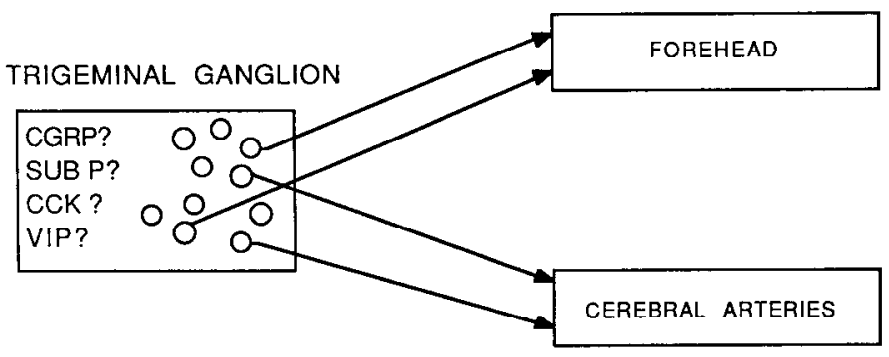

Figure 1. Schematic representation of the projections and neuropeptides investigated in these experiments. The proportions of neurons containing CGRP, CCK, VIP, and Sub P as well as TH and dynorphin, were studied in whole trigeminal ganglion and mandibular branch, and in the populations projecting to the cerebral arteries and forehead.

these Sub P and CGRP-containing fibers occurs after trigeminal ganglion lesions (Liu-Chen et al., 1983; Wanaka et al., 1986).

The occurrence and actions of Sub P on cerebral blood vessels has led to a model postulating Sub $P$ as critical in the vasodilation and pain propagation observed during migraine (Moskowitz, 1984) and cluster headaches (Hardebo, 1984). CGRP has also been observed in Sub P containing and in non-SPcontaining trigeminal ganglion cells (Lee et al., 1985a) and processes innervating the large cerebral arteries (Hanko et al., 1985). Recently, CGRP has been shown to be a potent vasodilator of cerebral arteries (Hanko et al., 1985; McCulloch et al., 1986). In facl, CGRP appears to be the most potent vasodilator of cerebral arteries (McCulloch et al., 1986), as compared with vasodilation mediated by other peptides, including VIP (Bevan et al., 1986). CGRP may also be involved in maintaining adequate blood perfusion to the cerebral cortex during chronic periods of vasoconstriction (McCulloch et al., 1986). Thus, CGRP may play a more important role than Sub $\mathrm{P}$ in the normal and perturbed vasomotor tone of intracranial vessels.

In the present study we sought to answer the following questions (Fig. 1): (1) Is the trigeminal innervation of the intracranial arteries enriched in a particular neuropeptide? (2) Are neuropeptides colocalized in cells that innervate the intracranial arteries? (3) What is the frequency of colocalization of neuropeptides throughout the trigeminal ganlion and in some of its other (nonintracranial vasculature) projections? A preliminary report of some of our findings has been presented elsewhere ( $\mathrm{O}^{\prime} \mathrm{Connor}$ and van der Kooy, 1986c).

\section{Materials and Methods}

Surgery. Adult male Charles River rats were anesthetized with intraperitoneal sodium pentobarbital (Somnotol, $65 \mathrm{mg} / \mathrm{kg}$ ). The fluorescent retrograde tracer employed was True blue (TB; obtained from Dr. Illing, FRG) dissolved in distilled water.

The middle cerebral artery or middle meningeal artery was isolated from a parietal-temporal approach. Preliminary immunocytochemical results were similar when expressed as a function of trigeminal ganglion cells retrogradely labeled from either artery, and thus the middle meningeal artery was used most often because of easier surgical access. After isolation of the artery, the dura and/or pia arachnoid on both sides was cut and a triangular piece of parafilm ( $3 \mathrm{~mm}$ high $\times 2 \mathrm{~mm}$ base) was pulled underneath the artery. Viscous $(>5 \%)$ TB was applied directly to the artery using a microsyringe or a wooden toothpick. The TB was allowed to adhere to the outer adventitia. The artery was then covered by 2 or 3 layers of gelfoam and another outermost layer of parafilm. The cut muscle and skin were sutured, and the animal was allowed to recover.

In a separate group of animals, TB was applied to the frontal branch of the ophthalmic nerve. The nerve was dissected free from tissue as it exited the supraorbital foramen and cut. TB soaked gelfoam was applied to the proximal stump of the nerve, and then covered by 2 additional layers of gelfoam and a strip of parafilm. The skin was sutured and animals were allowed to recover.

Combination of fluorescent retrograde tracing in vivo and immunocytochemistry in cultured trigeminal ganglia. To investigate the presence of various peptides in ganglion cells we used a novel method of combining in vivo fluorescent retrograde tracing with immunohistochemistry after explant culture of the trigeminal ganlion in colchicine-containing medium (Gibbins et al., 1985). This technique allows one to raise the neuropeptide levels in trigeminal perikarya with direct colchicine application, a difficult task in vivo. Briefly, $4 \mathrm{~d}$ following an application of TB to an intracranial artery, rats were decapitated, and the trigeminal ganglia were rapidly dissected out, washed in serum free medium, and then placed in serum-free medium containing $10^{-4} \mathrm{M}$ colchicine (Gibbins et al., 1985). Serum-free medium is composed of $80 \%$ Dulbecco's modified Eagle's medium (MEM) and 20\% Ham's F-12, supplemented with the following: non-essential amino acids ( $1 \%$ final concentration), $5 \mu \mathrm{g} / \mathrm{ml}$ insulin, $25 \mu \mathrm{g} / \mathrm{ml}$ transferrin, $1 \mu \mathrm{g} / \mathrm{ml}$ fibronectin, and $0.1 \mu \mathrm{M}$ selenium. Nine to $12 \mathrm{hr}$ later ganglia were immersed in $4 \%$ paraformaldehyde $\left(4^{\circ} \mathrm{C}\right)$ for $24 \mathrm{hr}$. Ganglia were then immersed in $20 \%$ sucrose $\left(4^{\circ} \mathrm{C}\right)$ overnight, and $32 \mu \mathrm{m}$ sections were cut in a cryostat. Sections were collected in $0.1 \mathrm{M}$ PBS $\left(4^{\circ} \mathrm{C}\right)$ containing $1.5 \%$ normal goat serum and $0.3 \%$ Triton $\mathrm{X}-100$.

Immunocytochemistry. Trigeminal ganglia sections were incubated with primary antisera to the following neuropeptides at $4^{\circ} \mathrm{C}$ overnight (24 hr): Sub P, CGRP, CCK, vasoactive intestinal polypeptide (VIP), tyrosine hydroxylase (TH), or dynorphin. All antisera were polyclonal, obtained from Immunonuclear Inc., except for the CGRP antiserum, which was a kind gift from Dr. Ron Evans (Salk Institute). The antisera were diluted (Sub P, 1:400; CGRP, 1:400: CCK, 1:300; VIP, 1:300; TH, 1:200; dynorphin, 1:200) in $0.1 \mathrm{M}$ PBS containing $1.5 \%$ normal goat serum and $0.3 \%$ Triton. After primary incubation the ganglia were rinsed 3 times (rinsing solution: $0.1 \mathrm{M}$ PBS containing $1.5 \%$ normal goat serum and $0.3 \%$ Triton $\mathrm{X}-100$ ) and then incubated for $60 \mathrm{~min}$ at room temperature with goat anti-rabbit IgG conjugated with fluorscein isothiocyanate (FITC) (Cappel) diluted 1:50. After a final 3 rinses in $0.1 \mathrm{M}$ PBS the ganglia were mounted onto gelation-coated slides and coverslipped with glycerol and water $(1: 1)$.

Double immunostaining. Ganglia sections to be double stained were incubated in an antisera cocktail containing both a Sub P monoclonal antiserum (Sera Lab) and the CGRP or CCK polyclonal antisera. The Sub P monoclonal was diluted 1:50 and the CCK or CGRP polyclonal at 1:200. Sections were incubated in the antisera cocktail overnight at $4^{\circ} \mathrm{C}$ and then rinsed 3 times in the rinsing solution described above, before being incubated in a biotinylated horse anti-mouse IgG (Cappel) for $60 \mathrm{~min}$ at room temperature. In an earlier study we found that some commercially available goat anti-rat IgG cross-react with rabbit polyclonal sera; however, a horse anti-mouse did not cross-react with the rabbit polyclonal sera but did react strongly with rat monoclonal sera (Miceli et al., 1987). After another 3 rinses in the rinsing solution, the sections were incubated in avidin-conjugated rhodamine isothiocyanate (1:50) for $60 \mathrm{~min}$ at room temperature. Following another 3 rinses in the rinsing solution, the sections were incubated with goat anti-rabbit FITC (1:50). After a final 3 rinses (in $0.1 \mathrm{~m}$ PBS), ganglia sections were mounted and coverslipped as described above.

All tissues were examined with an epifluorescent Leitz microscope. In the double-stained tissue, Sub $\mathrm{P}$ immunoreactivity appeared bright red when examined under a 550-nm-wavelength filter, while CGRP or CCK immunoreactivity appeared green under a 470-nm-wavelength filter. TB labeled the cytoplasm of cells and was visible as a deep blue label under a 390-nm-wavelength filter.

Reports of the proportion of CGRP-containing cells in the trigeminal ganglion have ranged from 1-2\% (Rosenfeld et al., 1983, 1984) to $40-$ $50 \%$ (Terenghi et al., 1985). Thus, we considered that a careful examination of the proportion of immunoreactive neurons was necessary. Sections through the mandibular branch and through the entire trigeminal ganglion were counted for immunoreactive neurons under fluorescent microscopy. The same sections were then Nissl-stained, and all the neurons (both labeled and unlabeled) in the appropriate whole ganglia section or mandibular branch section were counted.

Cross-reactivity control experiments. In order to assess the cross-reactivity of the primary antisera, ganglia sections were incubated with each antiserum absorbed with each of the following peptides (at $100 \mu \mathrm{g} / \mathrm{ml}$ ): CCK-8, Sub P (both from Sigma), and CGRP ${ }_{27-38}$ (Peninsula). The CGRP antiserum was produced in a rabbit against Tyr-CGRP $P_{23-38}$. A second set of experiments was employed to test the specificity of the secondary antisera. Ganglia sections incubated in CGRP and CCK rabbit poly- 

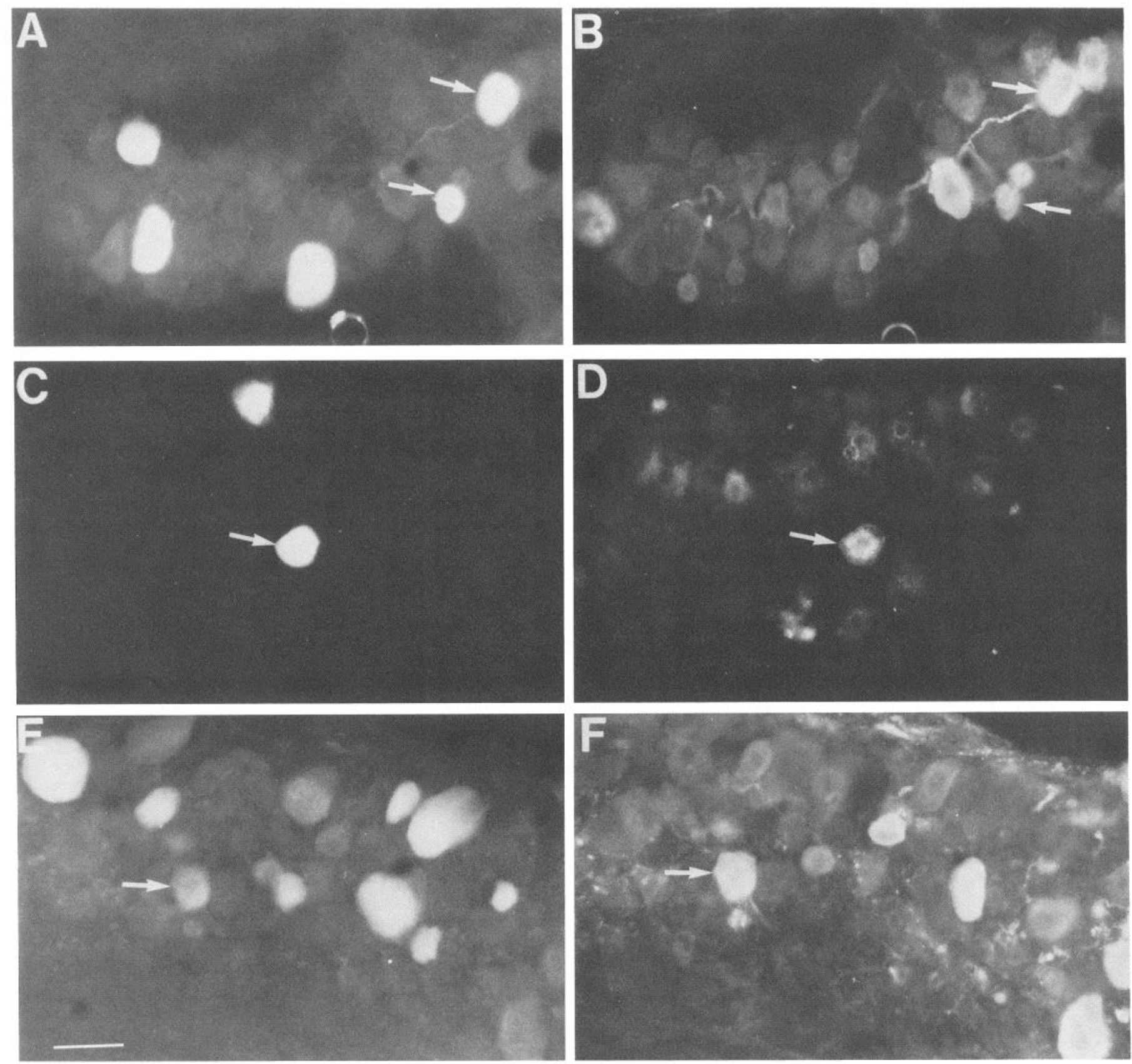

Figure 2. Sub P, CCK, or CGRP immunostaining in trigeminal ganglion cells retrogradely labeled with TB transported from the middle cerebral artery or forehead. $A-D$, Colocalization of Sub P or CCK, with TB retrogradely transported from the middle cerebral artery $A$ and $B$, TB and Sub P, respectively; $C$ and $D$, TB and CCK, respectively. $E$ and $F$, Colocalization of TB transported from the forehead $(E)$ and CGRP immunocytochemistry $(F)$. Each pair of photomicrographs $(A$ and $B, C$ and $D, E$ and $F$ ) shows the same field of the same section illuminated under excitation length of $360 \mathrm{~nm}(A, C, E$,) to show TB labeling and under a wavelength of $470 \mathrm{~nm}(B, D, F$, ) to show FITC labeling. Single ganglion cells containing both TB and a neuropeptide are indicated with an arrow in the $A$ and $B, C$ and $D$, and $E$ and $F$ pairs. Scale bar, $50 \mu \mathrm{m}$.

clonal primary antisera were then incubated in biotinylated horse antimouse IgG and then avidin-RITC to test for cross-reactivity. Sections that had incubated in Sub P mouse monoclonal antiserum were incubated with goat anti-rabbit FITC.

\section{Results}

\section{Immunocytochemistry and retrograde tracing}

Numerous cells were immunoreactive for CGRP, Sub P, and CCK in the trigeminal ganglia (Fig. 2). Antisera to VIP indicated the presence of a small population of cells (10-30/ganglion), whereas cells immunoreactive for dynorphin or $\mathrm{TH}$ were not observed. Pericellular arborizations (Katz and Karten, 1980;
Kuwayama and Stone, 1986) containing CGRP, Sub P, and CCK were also observed throughout the ganglion (not shown).

In general, immunocytochemical results from cultured ganglia were much better than those from noncultured ganglia. The superior results observed after culturing in colchicine appear to be due to the depolymerization of microtubules and consequent loss of peptide transport rather than to nonspecific culturing effects, for 2 reasons. First, there was no increase in nonspecific primary antibody labeling, as no cells were observed to stain for dynorphin or $\mathrm{TH}$ in the presence or absence of colchicine. TH (Price and Mudge, 1983) and dynorphin (Sweetnam et al., 


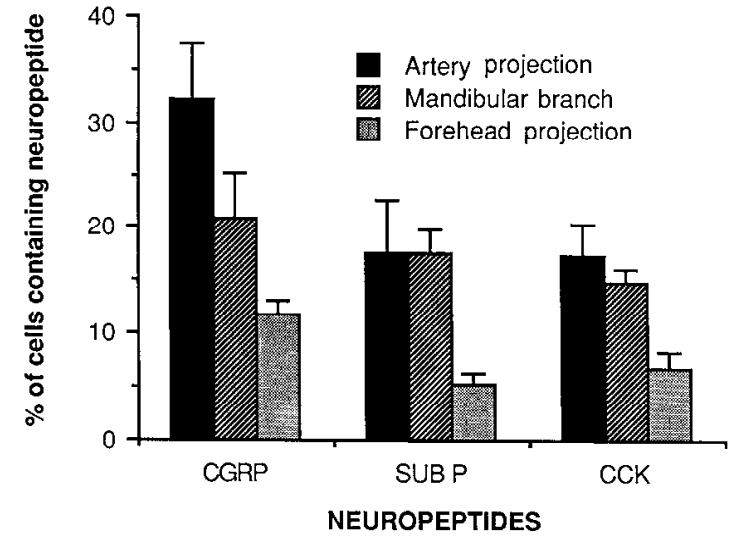

Figure 3. Comparison of CCK, Sub P, and CGRP neuropeptide immunocytochemical localization in cells throughout the mandibular branch of the trigeminal ganglion, in cells projecting to the cerebral vessels, or in cells projecting to the forchead. Frequencies (means \pm SEM) of all 3 neuropeptides are greater in the artery projecting cells than in the forehead projecting cells. However, only the percentage of CGRP-immunoreactive neurons is enriched in the vascular projecting cells when compared to cells throughout the mandibular branch of the ganglion. For mandibular counts a total of 8 sections from 3 animals were counted; total Nissl-stained cells counted: for CCK comparisons, $n=2652$; CGRP, $n=2602$; and Sub P, $n=2251$. For forehead counts all TB-labeled cells from 7 animals were counted. Total TB cells counted: for CCK comparisons, $n=1952$; CGRP, $n=1610$; and Sub P, $n=2341$. For artery counts all TB-labeled cells were again counted; for CCK comparisons ( 6 animals), $n=95$; CGRP (11 animals), $n=117$; and Sub P (11 animals), $n=182$.

1982) have been observed in small populations of dorsal root ganglion (DRG) cells. However, neither has previously been reported to be present in trigeminal ganglion cells. Second, the CGRP results did not differ significantly in cultured and noncultured ganglia. In cultured ganglia, the numbers of CGRPpositive cells did not increase significantly; however, more nerve processes and much brighter immunofluorescence of the cells were observed.

The distribution of ganglion cells projecting to intracranial arteries or to the forehead has previously been detailed (O'Connor and van der Kooy, 1986a), and the present retrograde tracing results replicate these previous findings. The most striking result of the present report is that the percentage of CGRP-containing cells $(32 \%)$ in the population of trigeminal ganglion cells projecting to the intracranial arteries is enriched compared with the percentage of CGRP-containing neurons observed in the population of cells projecting to the forehead $(12 \%)$ or compared with the population of CGRP-containing cells throughout the mandibular branch (21\%) (Fig. 3). Trigeminal cells innervating the intracranial arteries and forehead are separate populations found predominantly in the ophthalmic-maxillary branch of the adult ganglion (O'Connor and van der Kooy, 1986a). Thus, a comparison of these populations and the cells in the mandibular branch provides a wide sampling of trigeminal sensory cells. When similar comparisons are made for Sub P or CCK-containing neurons, the proportion of these peptides in the artery projecting populations reflects that observed throughout the mandibular branch (Fig. 3).

The enrichment of CGRP-containing neurons (32\%) in the artery projecting population is further demonstrated compared with the observed frequency of CGRP-containing cells in the whole ganglion (23\%; Fig. 4). However, the artery projection is not enriched with cells containing CCK (17\%) or Sub P (17.5\%)

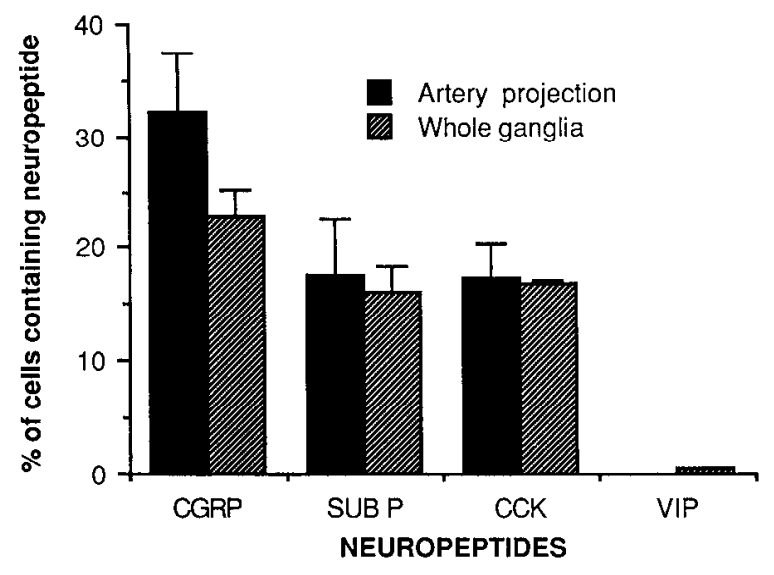

Figure 4. Comparison of CCK, Sub P, VIP and CGRP neuropeptide immunostaining in the trigeminal cells projecting to the cerebral vasculature compared with the immunostaining throughout the entire trigeminal ganglia. Only the number of CGRP-containing cells is enriched in the projection to the intracranial arteries. Less than $1 \%$ of trigeminal cells were immunoreactive with VIP, and none of these VIP-immunoreactive cells projected to the vasculature. TH and dynorphin immunoreactivity was not observed in any cells in the ganglia. (For whole ganglia counts a total of 8 complete sections were counted from 3 animals; total Nissl-stained cells counted for CCK comparisons, $n=12,056$; CGRP, $n=12,913$; and Sub P, $n=12,136$.)

compared with the whole ganglion (17\% CCK- and $16 \%$ Sub P-containing cells; Fig. 4). Interestingly, the proportion of CGRP, Sub $P$, and CCK-immunoreactive neurons in the mandibular branch reflects that observed in the whole ganglion (compare Figs. 3 and 4), whereas the observed proportion of cells immunoreactive for all 3 peptides in the forehead projection appears to be disproportionately low when compared with whole ganglia or the mandibular branch (Figs. 3, 4).

\section{Double immunostaining and retrograde tracing}

Sub $\mathrm{P}$ has been reported to coexist with CGRP in a population of trigeminal ganglion neurons (Lee et al., 1985a, b; Skofitsch and Jacobowitz, 1985; Wanaka et al., 1986). There is considerable variance between these reports concerning the proportion of CGRP neurons that also contain Sub P. Percentages of CGRP neurons also containing Sub P ranged from 50 to $90 \%$ in trigeminal and dorsal root ganglia (Gibbins et al., 1985; Lec ct al., 1985a, b; Wanaka et al., 1986). All these reports, however, indicated that there must also be a population of trigeminal ganglion cells that contained CGRP and not Sub P. Was this "CGRP only" population responsible for the observed CGRP enrichment in the intracranial artery projections? A rat monoclonal antibody to Sub $P$ was employed to enable double staining of trigeminal cells for coexistence of Sub P and CGRP (Fig. 5) or Sub $\mathrm{P}$ and CCK. Figure $6 A$ demonstrates that the enrichment of CGRP-containing neurons in the artery projection is due to the large number of CGRP-only-containing neurons (i.e., CGRP cells that do not have coexisting Sub P). Cells containing CGRP and not Sub $P$ made up $21 \%$ of the intracranial artery projecting population but only $9 \%$ of cells throughout the ganglia, whereas cells containing both CGRP and Sub P made up approximately the same percentage of cells in the artery projection (17\%) and in the whole ganglia (14\%). Very few neurons containing Sub $\mathrm{P}$ and not CGRP (Fig. 6A) or containing CCK and not Sub P (Fig. $6 B$ ) were observed in the vascular projection or throughout the ganglia. 

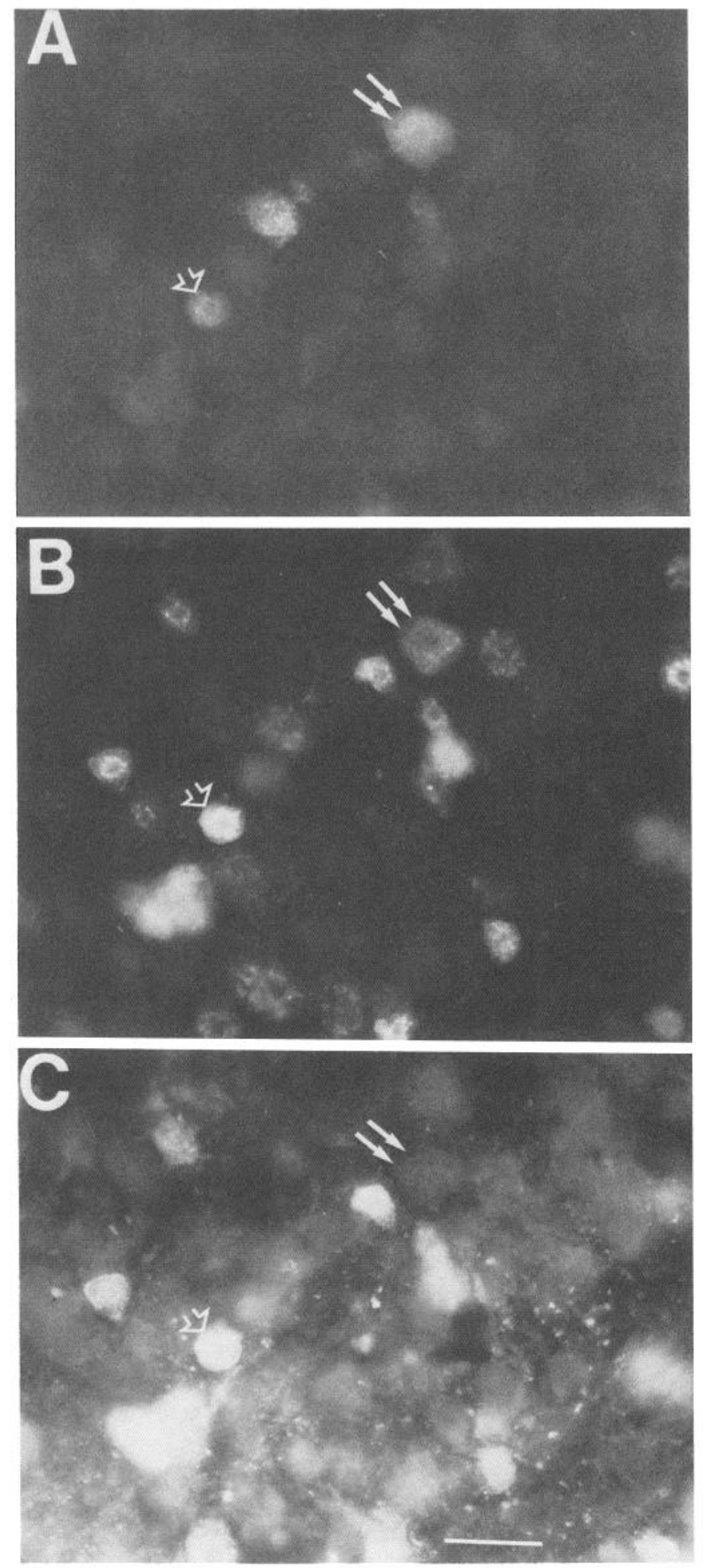

Figure 5. Colocalization of CGRP and Sub P immunostaining in trigeminal ganglion cells retrogradely labeled with TB transported from the middle meningeal artery. Photomicrographs of the same section illuminated under different excitation wavelengths to reveal cells retrogradely labeled with TB $(A)$, CGRP immunoreactivity $(B)$, and Sub $\mathrm{P}$ immunoreactivity $(C)$. A cell containing TB and both neuropeptides is indicated with an open arrow, while a TB cell containing ony CGRP is indicated with a double arrow. Scale bar, $50 \mu \mathrm{m}$.
Table 1. Neuropeptide colocalization in trigeminal ganglion

Cell type Percentage

CGRP cells containing SUB P

Sub $P$ cells containing CGRP

$60 \pm 2.5$

CCK cells containing SUB P

$90 \pm 2.8$

Sub $P$ cells containing CCK

$90 \pm 4.6$

$78 \pm 5.3$

Comparison of CGRP and Sub P colocalization and CCK and Sub P colocalization throughout the trigeminal ganglion. CGRP is colocalized in $90 \%$ of Sub P cells, and Sub P is colocalized in $90 \%$ of CCK cells. Thus, a major portion of all CCKcontaining neurons would be predicted to also contain CGRP and Sub P. Means $( \pm$ SEM) for CGRP and Sub P colocalization were from 5 animals; $n=560$ for each of Sub P immunoreactive neurons and CGRP-immunoreactive neurons. Counts for CCK and Sub P colocalization were from 4 animals; $n=315$ for Sub $\mathrm{P}$ immunoreactive neurons and 320 for CCK-immunoreactive neurons.

Most of the Sub P and CCK projections to the intracranial arteries come from cells that contained both Sub P and CCK, although a notable proportion of cells projecting to the arteries contains Sub P without CCK (11\%). This does not reflect an enrichment of Sub P, however, as most of these neurons also contain CGRP (Fig. 6A). Thus, the notable proportion of Sub $\mathrm{P}$ (and not $\mathrm{CCK}$ ) containing neurons with artery projections may be an artifact of the large number of neurons containing CGRP and Sub P. Approximately $95 \%$ of CCK cells projecting to the artery (Fig. $6 B$ ) and $90 \%$ throughout the ganglia (Table 1) were observed to contain Sub $P$ also. These data predict that a population of artery innervating trigeminal cells (and indeed a population throughout the ganglia) contain all 3 neuropeptides. Given that $90 \%$ of CCK cells in the ganglia also contain Sub P (Table 1) and that $90.6 \%$ of these Sub P cells also contain CGRP (Table 1), then the majority of CCK-containing cells in the ganglia should also contain both Sub P and CGRP. This provides indirect evidence for the trilocalization of neuropeptides in single trigeminal sensory neurons.

In all of the experiments reported here, the absolute numbers of labeled cells may be underestimated by immunocytochemistry and retrograde labeling techniques. Nevertheless, the relative comparisons that are made should be valid in all cases.

\section{Cross-reactivity control experiments}

Only when each of the antisera was adsorbed by the specific antigen it was raised against did we observe no immunoreactive positive staining. In the second set of control experiments, we found no cross-reactivity of the goat anti-rabbit secondary antiserum with horse anti-mouse biotinylated secondary or with rat monoclonal primary antisera. There was also no cross-reactivity of the horse anti-mouse biotinylated secondary or avidin-RITC with the rabbit primary antisera.

\section{Discussion}

The sensory trigeminal innervation of the intracranial cerebral arteries contains a disproportionately large number of CGRPimmunoreactive neurons. Approximately $32 \%$ of the trigeminal cells that are sensory to the intracranial arteries contain CGRP. In contrast, only $12 \%$ of the neurons innervating the forehead, $21 \%$ of the neurons localized in the mandibular branch, or $23 \%$ of neurons throughout the ganglia contain CGRP. This disproportionately high frequency of CGRP neurons in the vascular projection is further illustrated in comparisons with the frequencies of other neuropeptides in trigeminal ganglion neurons. Both Sub P and CCK have been observed on the walls of cerebral 
A
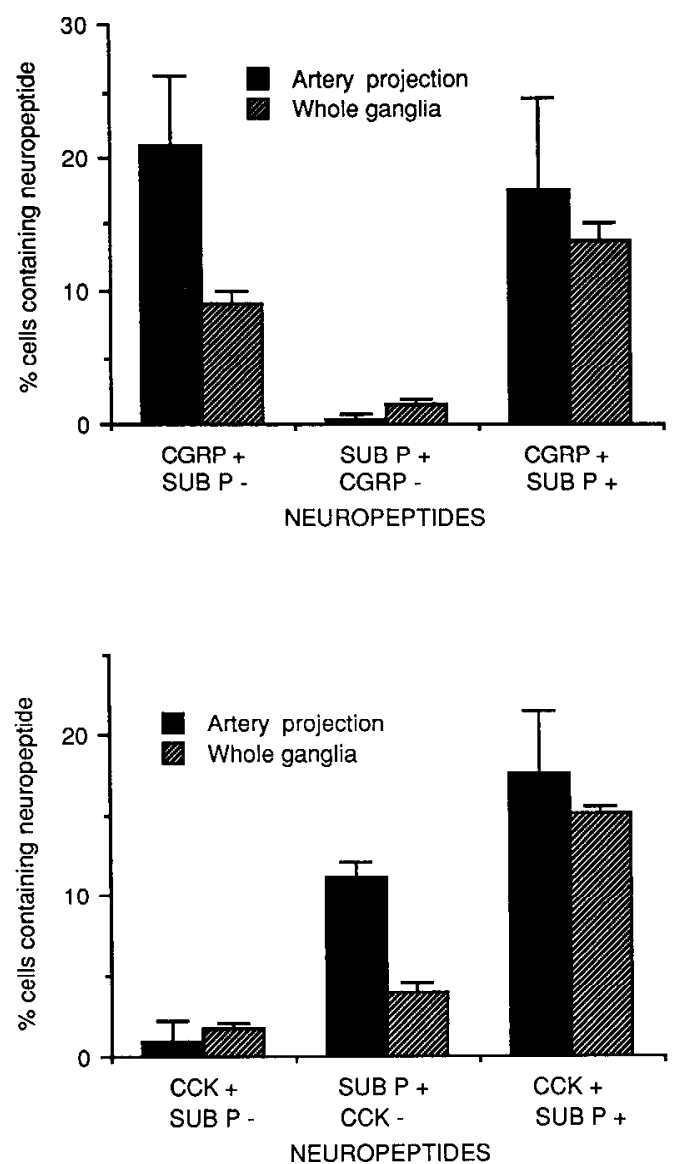

Figure 6. Colocalization of CGRP and Sub P, or CCK and Sub P. Comparison of individual cells containing CGRP but not Sub P, Sub $\mathrm{P}$ but not CGRP, and both CGRP and Sub $\mathrm{P}(A)$ and containing CCK but not Sub P, Sub P but not CCK, and both CCK and Sub $\mathrm{P}(B)$ in the population projecting to the cerebral arteries or in the population throughout the ganglia. More than half of the CGRP vascular projection is composed of cells containing CGRP but not Sub P. The majority of Sub $P$ cells that project to the vasculature also contain CCK. For artery counts, all TB-labeled cells were counted: for CGRP/Sub P comparisons (4 animals), $n=105$; CCK/Sub P ( 3 animals), $n=51$. Ganglion percentages were derived from applying the results in Table 1 to whole ganglion counts in Figure 3. For example, 23\% of the neurons in the whole ganglion contain CGRP. From Table 1, $60 \%$ of these cells also contain Sub P. Thus, in the whole ganglion, approximately $14 \%$ of CGRP-positive cells also contain Sub P.

arteries, and the major source of these fibers appears to be the trigeminal ganglion (Liu-Chen et al., 1984, 1985, 1986; Wanaka et al., 1986; the present results). We found, however, that the proportion of artery projecting cells that are immunoreactivc for CCK or Sub P did not differ from the proportions observed in the mandibular branch or throughout the ganglion. Interestingly, the frequency of all 3 neuropeptides was disproportionately low in the forehead projecting cells compared with the frequency in the mandibular branch or throughout the ganglia. Thus, CGRP is enriched in the vascular projection with respect to its observed frequency throughout the ganglia, and with respect to the observed frequency of other neuropeptides contained in this and other trigeminal projections.

The coexistence of Sub P and CGRP in single neurons in dorsal root and trigeminal ganglia is well established (Gibbins et al., 1985; Lee et al., 1985a, b; Skofitsch and Jacobowitz, 1985;
Wanaka et al., 1986). We found that most Sub P cells (90\%) also contain CGRP, as observed by others (Lee et al., 1985a, b; Wanaka et al., 1986), and that about $60 \%$ of the CGRPpositive cells also contain Sub P. Given our result that CGRP, but not Sub $\mathrm{P}$, is enriched in the trigeminal projections to the intracranial arteries, it seemed likely that enrichment was accounted for by cells containing only CGRP (and not Sub P). This prediction was confirmed in the double-immunostaining studies which revealed that cells containing CGRP and not Sub $P$ make up $21 \%$ of the population projecting to the intracranial arteries but only $9 \%$ of cells throughout the whole ganglia. Thus, the enrichment of CGRP in the vasculature projection appears to be due to a disproportionate number of CGRP-postive neurons that do not contain Sub P.

A small population of VIP-immunoreactive cells was also observed in the trigeminal ganglion. None of these cells projected to the forehead or intracranial arteries. Thus, it is most probable that the majority of the VIP innervation of the intracranial arteries is derived from parasympathetic projections from the sphenopalatine ganglion, neurons that also contain $\mathrm{ACh}$ (Hara et al., 1985). The overall frequency of VIP neurons in the trigeminal ganglion was less than $1 \%$. This frequency of VIP neurons is much lower than that reported in the guinea pig trigeminal ganglion (Kummer and Heym, 1986). Cells immunoreactive for TH and dynorphin were not observed in the trigeminal ganglion.

The existence of CCK-containing fibers on cerebral arteries was demonstrated by Liu-Chen et al. (1985), and it was proposed that the trigeminal ganglion was the source of these fibers. We have shown directly that approximately $18 \%$ of the neurons projecting to the cerebral arteries contain $\mathrm{CCK}$. The coexistence of CCK with Sub P has been demonstrated in DRG (Dalsgaard et al., 1982; Tuchscherer and Seybold, 1985) and more recently in the guinea pig trigeminal ganglia (Kuwayama et al., 1987). We found in the artery projection and throughout the whole ganglia that virtually all $(>90 \%)$ of the CCK-immunoreactive cells also contain Sub P. The high frequency of CGRP (>90\%) in Sub $P$ positive trigeminal ganglion cells further suggests a trilocalization of CGRP, Sub P, and CCK in many ganglion neurons.

At present, no general functional role for CGRP in the sensory nervous system has been posited, although anatomically it appears to be enriched in noncutaneous (primarily visceral) sensory neurons (Su et al., 1986; Green and Dochray, 1987; present results). CGRP enrichment has been observed in sensory projections to the upper gastrointestinal tract (Green and Dochray, 1987), urinary tract, and kidney (Su et al., 1986). CGRP enrichment has also been reported in trigeminal projections to the tongue (Terenghi et al., 1986). We have also observed CGRP enrichment in trigeminal-tongue projections, although we find the enrichment of cells containing CGRP and not Sub P to be less than in trigeminovascular projections (unpublished observations). Indeed, the tongue enrichment appears to be due to neurons that contain both CGRP and Sub P (unpublished observations). Kuwayama et al. (1987) found no enrichment of CGRP, Sub P, or CCK in the trigeminal projection to the eye, and we find no enrichment (in fact, a paucity) of these neuropeptides in trigeminal projections to the forehead. Although not emphasized by the authors (Su et al., 1986; Green and Dochray, 1987), a major portion of the CGRP enrichment in the upper gastrointestinal tract and urinary tract in the rat is due to a population of CGRP cells that did not contain Sub P. Thus, a 
general principle emerges in that sensory projections to visceral targets are enriched in CGRP and, moreover, that a major portion of these projections in rats appears to be due to a population of cells that contain CGRP but not Sub P (Su et al., 1986; Green and Dochray, 1987; present results).

Functionally, the CGRP, Sub P, and CCK contained in the trigeminovascular projection may be important for the mechanisms of migraine headache (Moskowitz, 1984) and/or in controlling vasomotor tone (Edvinsson et al., 1981; McCulloch et al., 1986). Other neurotransmitters are also undoubtedly involved. The cerebral arteries are also innervated by VIP-containing (Hara et al., 1985; Matsuyama et al., 1985; Bevan et al., 1986), serotonergic (Edvinsson et al., 1983; Marco et al., 1985), neuropeptide Y-containing (Edvinsson et al., 1983; Matsuyama et al., 1985), and catecholaminergic (Matsuyama et al., 1985) fibers. However, there is no coherent functional explanation for the neurochemical diversity of the innervation of the cerebral vasculature. The pathways containing these neurotransmitters have been postulated either to vasoconstrict or to dilate pial artcries, and thus possibly influence cerebral blood flow. All of the peptides (CGRP, Sub P, CCK, VIP) studied in the present report have been shown to vasodilate cerebral arteries to varying degrees (Edvinsson et al., 1981; Zawadzki et al., 1983; Bevan et al., 1986; McCulloch et al., 1986), with the most potent vasodilator of arteries in vivo and in vitro being CGRP (McCulloch et al., 1986). Interestingly, CGRP also appears to augment the release of peptides from primary afferents centrally (Oku et al., 1987). If this presynaptic facilitation also occurs peripherally on primary afferents, then activated CGRP fibers may positively regulate the release of additional CGRP (as well as Sub P) peripherally along the blood vessels. Trigeminal sensory fibers containing Sub $\mathbf{P}$, and presumably CGRP, have been observed in the adventia and along the advential-media border (but not within the media or the endothelium) of feline cerebral arteries (Liu-Chen et al., 1986). Thus, a local augmentation of CGRP and Sub $P$ release along the vesscls may bc an integral part of the proposed axon reflex mechanism for vasodilation observed during migraine headaches (Moskowitz, 1984). This facilitative influence on peptide release by CGRP may also be helpful in antagonizing excess arterial vasoconstriction in conditions such as subarachnoid hemorrhage (McCulloch et al., 1986).

The CGRP-induced spinal cord release of peptides from primary afferents (most notably the release of Sub P) also decreases the threshold for spinal dorsal horn activation by a noxious mechanical stimulus (Oku et al., 1987). This effect may be dependent on presynaptic CGRP receptors on Sub P afferent endings and on an accompanying peripheral stimulus, as intrathecal CGRP administration alone has very little noxious effect (Oku et al., 1987). Perhaps the enrichment of CGRP in the trigeminovasculature projecting neurons may serve to enhance the debilitating pain originating from an intracranial stimulus. The central release of CGRP may also directly stimulate other central primary afferents in the dorsal horn that are sensory to cutaneous targets, thus resulting in referred pain.

The enrichment of CGRP in the trigeminovascular projection and the disproportionately low frequency of all the neuropeptides examined in the trigeminal forehead projection, raise the question of the mechanisms underlying target-regulated, phenotypic protein expression in neurons. There are 2 possible developmental scenarios. First, certain trigeminal cells may be committed early on to be CGRP-containing vascular innervating cells. These cells specifically project to vascular targets or specifically survive and are maintained on those targets. Second, trigeminal neurons may be unspecialized until they reach their targets, at which time there is a target-induced phenotypic expression in the neurons. There are numerous reports of environmentally regulated (see reviews by Patterson, 1978, and Black et al., 1987), and more specifically target-induced (McMahon and Gibson, 1987; Smith and Frank, 1987), phenotypic expression. Central connections (Smith and Frank, 1987) and neurotransmitter expression (McMahon and Gibson, 1987) can be altered when neural afferents to skin and muscle are experimentally redirected to innervate inappropriate targets. However, our previous developmental studies may be taken as some support for the first developmental scenario. The adult pattern of trigeminal innervation of the intracranial arteries is produced only after a period of substantial postnatal cell death (O'Connor and van der Kooy, 1986a). It is tempting to speculate that only those artery cells specifically committed to CGRP expression survive postnatally to adulthood. However, even in this scenario it remains possible that the initial expression of CGRP in some early ganglion cells is target driven, as trigeminal projections reach their targets prenatally (Lumsden and Davies, 1983).

The trigeminal sensory projection to the cerebral arteries is enriched with the neuropeptide CGRP. This peptide may be important in the peripheral physiology and central transmission of changes in cerebral vasculature homeostasis. It will be important to determine the possible roles of target-regulated synthesis or preferential cell death of non-CGRP-containing neurons as mechanisms underlying the neuropeptide enrichment observed in the adult.

\section{References}

Bevan, J. A., G. M. Guga, M. A. Moskowitz, and S. I. Said (1986) In vitro evidence that vasoactive intestinal peptide is a transmitter of neuro-vasodilatation in the head of the cat. Neuroscience 19: 597604

Black, I. B., J. E. Adler, C. F. Dreyfus, W. F. Friedman, E. F. Lagamma, and A. H. Roach (1987) Biochemistry of information storage in the ncrvous systcm. Sciencc 236: 1263-1268.

Borges, L. F., and M. A. Moskowitz (1983) Do intracranial and extracranial trigeminal afferents represent divergent collaterals? Neurosci. Lett. 35: 265-270.

Dalsgaard, C.-J., S. R. Vincent, T. Hökfelt, J. M. Lundberg, A. Dahlstrom, M. Schultzberg, G. J. Dockray, and A. C. Cuello (1982) Coexistence of cholecystokinin-and substance P-like peptides in neurons of the dorsal root ganglia of the rat. Neurosci. Lett. 33: 159-163.

Davis, K. D., and J. O. Dostrovsky (1986) Activation of trigeminal brain-stem nociceptive neurons by dural artery stimulation. Pain 25 . $395-401$

Edvinsson, L., J. McCulloch, and R. Uddman (1981) Substance P: Immunohistochemical localization and effect upon cat pial arteries in vitro and in situ. J. Physiol. (Lond.) 318: 251-258.

Edvinsson, L., P. Emson, J. McCulloch, K. Tatemoto, and R. Uddman (1983a) Neuropeptide Y: Cerebrovascular innervation and vasomotor effects in the cat. Neurosci. Lett. 43: 79-84.

Edvinsson, L., J. A. Degueurce, D. Duverger, E. T. MacKenzie, and B. Scatton (1983b) Central serotonergic nerves project to the pial vessels of the brain. Nature 306: 55-57.

Fay, T. (1932) Atypical facial neuralgia, a syndrome of vascular pain. Ann. Otol. 41: 1030-1062.

Gazelius, B., and L. Olgart (1980) Vasodilation in the dental pulp produced by electrical stimulation of the inferior alveolar nerve in the cat. Acta Physiol. Scand. 108: 181-186.

Gibbins, I. L., J. B. Furness, M. Costa, I. MacIntyre, C. J. Hillyard, and S. Girgis (1985) Colocalization of calcitonin gene-related peptide like immunoreactivity with substance $P$ in cutaneous, vascular, and visceral sensory neurons of guinca pigs. Ncurosci. Lett. 57: 125-130. Goadsby, P. J., G. A. Lambert, and J. W. Lance (1986) Stimulation 
of the trigeminal ganglion increases flow in the extracerebral but not the cerebral circulation of the monkey. Brain Res. 381: 63-67.

Green, T., and G. J. Dochray (1987) Calcitonin gene-related peptide and substance $P$ in afferents to the upper gastrointestinal tract in the rat. Neurosci. Lett. 76: 151-156.

Hanko, J., J. E. Hardebo, J. Kahrstrom, C. Owman, and F. Sundler (1985) Calcitonin gene-related peptide is present in mammalian cerebrovascular nerve fibres and dilates pial and peripheral arteries. Neurosci. Lett. 57: 91-95.

Hara, H., G. S. Hamill, and D. M. Jacobowitz (1985) Origin of cholinergic nerves to the rat major cerebral arteries: Coexistence with vasoactive intestinal polypeptide. Brain Res. Bull. 14: 179-188.

Hardebo, J. E. (1984) The involvement of trigeminal substance $P$ neurons in cluster headache. An hypothesis. Headache 24: 294-304.

Katz, D. M., and H. J. Karten (1980) Substance $P$ in the vagal sensory ganglia: Localization in cell bodies and pericellular arborizations. J. Comp. Neurol. 193: 549-564.

Kummer, W., and C. Heym (1986) Correlation of neuronal size and peptide immunoreactivity in the guinea-pig trigeminal ganglion. Cell Tissue Res. 245: 657-665.

Kuwayama, Y., and R. A. Stone (1986) Neuropeptide immunoreactivity of pericellular baskets in the guinea pig trigeminal ganglion. Neurosci. Lett. 64: 169-172.

Kuwayama, Y., G. Terenghi, J. M. Polak, J. O. Trojanowski, and R. A Stone (1987) A quantitative correlation of substance P-, calcitonin gene-related peptide- and cholecystokinin-like immunoreactivity with retrogradely labeled trigeminal ganglion cells innervating the eye. Brain Res. 405: 220-226.

Lee, Y., Y. Kawai, S. Shiosaka, K. Takami, H. Kiyamam, C. J. Hillyard, S. Girgis, I. MacIntyre, P. C. Emson, and M. Tohyama (1985a) Coexistence of calcitonin gene-related peptide and substance P-like peptide in single cells of the trigeminal ganglion of the rat: Immunohistochemical analysis. Brain Res. 330: 194-196.

Lee, Y., K. Takami, Y. Kawai, S. Girgis, C. J. Hillyard, I. MacIntyre, P. C. Emson, and M. Tohyama (1985b) Distribution of calcitonin gene-related peptide in the rat peripheral nervous system with reference to its coexistence with substance P. Neuroscience 15: 12271237.

Liu-Chen, L.-Y., M. R. Mayberg, and M. A. Moskowitz (1983) Immunohistochemical evidence for a substance P-containing trigeminovascular pathway to pial arteries in cats. Brain Res. 268: 162-166.

Liu-Chen, L.-Y., S. A. Gellespie, T. V. Norregaard, and M. A. Moskowitz (1984) Co-localization of retrogradely transported wheat germ agglutinin and the putative neurotransmitter substance $\mathbf{P}$ within trigeminal ganglion cells projecting to cat middle cerebral artery. J. Comp. Neurol. 225: 187-192.

Liu-Chen, L.-Y., T. V. Norregaard, and M. A. Moskowitz (1985) Some cholecystokinin-8 immunoreactive fibers in large pial arteries originate from trigeminal ganglion. Brain Res. 359: 166-176.

Liu-Chen, L.-Y., T. M. Liszczak, J. C. King, and M. A. Moskowitz (1986) Immunoelectron microscopic study of substance P-containing fibers in feline cerebral arteries. Brain Res. 369: 12-20.

Lumsden, A. G. S., and A. M. Davies (1983) Earliest sensory nerve fibres are guided to peripheral targets by attractants other than nerve growth factor. Nature 306: 786-788.

Marco, E. J., G. Balfogon, M. Salaices, C. F. Sanchez-Ferreer, and J. Marin (1985) Serotonergic innervation of cat cerebral arteries. Brain Res. 338: 137-139.

Matsuyama, T., S. Shiosaka, A. Wanaka, S. Yoneda, K. Kimura, T. Hayakawa, P. C. Emson, and M. Tohyama (1985) Fine structure of peptidergic and catecholaminergic nerve fibers in the anterior cerebral artery and their interrelationship: An immunoelectron microscopic study. J. Comp. Neurol. 235: 268-276.

Mayberg, M. K., K. S. Langer, N. T. Zervas, and M. A. Moskowitz (1981) Perivascular meningeal projections from cat trigeminal ganglia: Possible pathway for vascular headaches in man. Science 213: 228-230.

Mayberg, M. R., N. T. Zervas, and M. A. Moskowitz (1984) Trigeminal projections to supratentorial pial and dural blood vessels in cats demonstrated by horseradish peroxidase histochemistry. J. Comp. Neurol. 223: 46-56.

McCulloch, J., R. Uddman, T. A. Kingman, and L. Edvinsson (1986) Calcitonin gene-related peptide: Functional role in cerebrovascular regulation. Proc. Natl. Acad. Sci. USA 83: 5731-5735.
McMahon, S. B., and S. Gibson (1987) Peptide expression is altered when afferent nerves reinnervate inappropriate tissue. Neurosci. Lett. 73:9-15.

McMahon, M., T. V. Norregaard, B. D. Beyerl, L. F. Borges, and M. A. Moskowitz (1985) Trigeminal afferents to cerebral arteries and forehead are not divergent axon collaterals in cat. Neurosci. Lett. 60 : 63-68.

Miceli, M. O., C. A. Post, and D. van der Kooy (1987) Catecholamine and serotonin colocalization in projection neurons of the area postrema. Brain Res. 412: 381-385.

Moskowitz, M. A. (1984) The neurobiology of vascular head pain. Ann. Neurol. 16: 157-168.

O'Connor, T. P., and D. van der Kooy (1986a) Pattern of intracranial and extracranial projections of trigeminal ganglion cells. J. Neurosci. 6: 2200-2207.

O'Connor, T. P., and D. van der Kooy (1986b) Cell death organizes the postnatal development of the trigeminal innervation of the cerebral vasculature. Dev. Brain Res. 27: 223-233.

O'Connor, T. P., and D. van der Kooy (1986c) Neuropeptides contained in the trigeminal projection to the cerebral vasculature Soc. Neurosci. Abstr. 12(1): 302.

Oku, R., M. Satoh, N. Fujii, A. Otaka, H. Yajima, and H. Takagi (1987) Calcitonin gene-related peptide promotes mechanical nociception by potentiating release of substance $P$ from the spinal dorsal horn in rats. Brain Res. 403: 350-354.

Olgart, L., B. Gazelius, E. Brodin, and G. Nilsson (1977) Release of substance P-like immunoreactivity from the dental pulp. Acta Physiol. Scand. 101: 510-512.

Patterson, P. (1978) Environmental determination of autonomic neurotransmitter functions. Annu. Rev. Neurosci. 1: 1-17.

Penfield, W., and F. McNaughton (1940) Dural headache and innervation of the dura mater. Arch. Neurol. Psychiatr. 44: 43-75.

Price, J., and A. W. Mudge (1983) A subpopulation of rat dorsal root ganglion neurons is catecholaminergic. Nature 301: 241-243.

Ray, B. S., and H. G. Wolff (1940) Experimental studies on headache. Arch. Surg. 41: 813-856.

Rosenfeld, M. G., J.-J. Mermod, S. G. Amara, L. W. Swanson, P. E. Sawchenko, J. Rivier, W. W. Vale, and R. H. Evans (1983) Production of a novel neuropeptide encoded by the calcitonin gene via tissue-specific RNA processing. Nature 304: 129-135.

Rosenfeld, M. G., S. G. Amara, and R. M. Evans (1984) Alternative RNA processing: Determining neuronal phenotype. Science 225 : 1315 1320.

Saito, K., L.-Y. Liu-Chen, and M. A. Moskowitz (1987) Substance Plike immunoreactivity in rat forebrain leptomeninges and cerebral vessels originates from the trigeminal but not sympathetic ganglia. Brain Res. 403: 66-71.

Skofitsch, G., and D. M. Jacobowitz (1985) Calcitonin gene-related peptide coexists with substance $P$ in capsaicin sensitive neurons and sensory ganglia of the rat. Peptides 6: 747-754.

Smith, C. L., and E. Frank (1987) Peripheral specification of sensory neurons transplanted to novel locations along the neuraxis. J. Neurosci. 7: 1537-1549.

Steiger, H. J., J. M. Tew Jr., and J. T. Keller (1982) The sensory representation of the dura mater in the trigeminal ganglion of the cat. Neurosci. Lett. 31: 231-236.

Strassman, A. G., P. Mason, M. A. Moskowitz, and R. Macieicz (1986) Response of brainstem trigeminal ncurons to clectrical stimulation of the dura. 379: 242-250.

Su, H. C., J. Whartron, J. M. Polak, P. K, Mulderry, M. A. Ghatei, S. J. Gibson, G. Terenghi, J. F. B. Morrison, J. Ballesta, and S. R. Bloom (1986) Calcitonin gene-related peptide immunoreactivity in afferent neurons supplying the urinary tract: Combined retrograde tracing and immunohistochemistry. Neuroscience 18: 727-747.

Sweetnam, P. M., J. H. Neale, J. L. Barker, and A. Goldstein (1982) Localization of immunoreactive dynorphin in neurons cultured from spinal cord and dorsal root ganglia. Proc. Natl. Acad. Sci. USA 79: 6742-6746.

Terenghi, G., J. M. Polak, M. A. Ghatei, P. K. Mulderry, J. M. Butler, W. G. Unger, and S. R. Bloom (1985) Distribution and origin of calcitonin gene-related peptide (CGRP) immunoreactivity in the sensory innervation of the mammalian cyc. J. Comp. Ncurol. 233: 506516.

Terenghi, G., J. M. Polak, J. Rodrigio, P. K. Mulderry, and S. R. Bloom 
(1986) Calcitonin gene-related peptide-immunoreactive nerves in the tongue, epiglottis and pharynx of the rat: Occurrence, distribution and origin. Brain Res. 365: 1-14.

Tuchscherer, M. M., and V.S. Seybold (1985) Immunohistochemical studies of substance $P$, cholecystoinin-octapeptide and somatostatin in dorsal root ganglia of the rat. Neuroscience 14: 593-605.

Wanaka, A., T. Matsuyamam, S. Yoneda, K. Kimura, T. Kamada, S. Girgis, I. MacIntyre, P. C. Emson, and M. Tohyamam (1986) Origins and distribution of calcitonin gene-related peptide-containing nerves in the wall of the cerebral arteries of the guinea pig with special reference to the coexistence with substance P. Brain Res. 369: 185192.

Wirth, F. P., and J. M. Van Buren (1971) Referral of pain from dural stimulation in man. J. Neurosurg. 34: 630-642.

Zawadzki, J. V., R. F. Furchgott, and P. D. Cherry (1983) Endothelium-dependent relaxation of arteries by octa-substance $P$, kassinin and octa-cholecystokinin. Fed. Proc. 42: 619. 